\title{
The challenges of estimating the impact of distributed energy resources flexibility on the TSO/DSO boundary node operating points
}

\author{
João Silva a,b, Jean Sumaili a,*, Ricardo J. Bessa ${ }^{a}$, Luís Seca ${ }^{a, b}$, Manuel Matosa,b, \\ Vladimiro Miranda ${ }^{\mathrm{a}, \mathrm{b}}$ \\ a INESC Technology and Science, INESC TEC, Rua Dr. Roberto Frias, 4200 Porto, Portugal \\ ${ }^{\mathrm{b}}$ Faculty of Engineering, University of Porto, FEUP, Porto, Portugal
}

\section{A R T I C L E I N F O}

\section{Article history:}

Received 10 February 2017

Revised 1 June 2017

Accepted 4 June 2017

Available online $\mathrm{xxx}$

\section{Keywords:}

Discrete variables

Distribution networks

Flexible assets

Optimization

Tangent straight lines

Transmission networks

\begin{abstract}
A B S T R A C T
The increasing penetration of renewable energy sources characterized by a high degree of variability and uncertainty is a complex challenge for network operators that are obligated to ensure their connection while keeping the quality and security of supply. In order to deal with this variable behavior and forecast uncertainty, the distribution networks are equipped with flexible distributed energy resources capable of adjusting their operating point to avoid technical issues (voltage problems, congestion, etc.). Within this paradigm, the flexibility that, in fact, can be provided by such resources, needs to be estimated/forecasted up to the transmission network node (primary substation) and requires new tools for TSO/DSO coordination. This paper addresses this topic by developing a methodology capable of finding the flexibility area while taking into account the technical grid constraints. The proposed approach is based on the formulation of a single optimization problem which is run several times, according with the expected precision for the flexibility area estimation. To each optimization problem run, a different objective function belonging to a family of straight lines is assigned. This allows exploring the active and reactive power flow limits at the TSO/DSO boundary nodes - which define the flexibility area. The effectiveness of the proposed model has been evaluated on two test networks and the results suggest a step forward in the TSO/DSO coordination field. Nevertheless, further investigations to study the effect of assets with discrete control nature (e.g., on load tap changers - OLTC, capacitor banks) on the occurrence of disjoint flexibility areas should be carried.
\end{abstract}

(c) 2017 Elsevier Ltd. All rights reserved.

\section{Introduction}

The increasing integration of variable and uncertain distributed renewable energy resources (DRES) induces a high level of complexity in order to balance supply and demand and handle local technical problems. Therefore, the variable nature and the forecast errors need to be effectively accommodated by the system operators without affecting the quality of supply standards. Challenges such as thermal unit cycling (Troy et al., 2010), thermal unit ramping insufficiencies and increased reserve requirements (Wan, 2011) are arising and their impact on the operation of transmission and distribution systems is expected to grow. The system needs to have the ability to react to sudden changes within acceptable time constraints.

\footnotetext{
* Corresponding author.

E-mail addresses: joao.v.silva@inesctec.pt (J. Silva), jean.sumaili@inesctec.pt

(J. Sumaili), ricardo.j.bessa@inesctec.pt (R.J. Bessa), lseca@inesctec.pt (L. Seca), mmatos@inesctec.pt (M. Matos), vmiranda@inesctec.pt (V. Miranda).
}

The flexible distributed energy resources (DER) that are connected to the distribution networks (e.g. controllable DRES, demand response, storage systems) can serve as a solution to these problems by providing different degrees of flexibility. In Eurelectric (2015), flexibility is defined as the modification of generation injection and/or consumption patterns in reaction to an external signal (price signal or activation) in order to provide a service to the system.

The range of flexibility services that can be offered to the system is spread through all timeframes (i.e. network and operational planning, real-time operations and ex-post) and past works already discussed and studied them. Within the planning domain, Nosair and Bouffard (2015) developed the concept of flexibility envelopes in order to analyze if the four typical reserve requirements can be met through the flexibility available, while Nosair and Bouffard (2016) focus on considering the operating reserve as deployable energy. The topic discussed in Nosair and Bouffard (2016) is very relevant since the fast variability introduced by DRES imposes the 
necessity of operating reserves with ample ramp rates that can be provided by some flexible DER such as storage systems.

Another important topic discussed in the literature is the provision of ancillary services. A model to aggregate the flexibility of thermostatically controlled loads (TCLs) is presented in Zhao and Zhang (2016) aiming to provide frequency regulation services. To model multi-period flexibility, Zhao and Zhang (2016) develops a geometric model that uses the Minkowski sum of individual polytopes and that aggregates multiple TCL. The inclusion of flexible resources in the transmission level dispatch is assessed in Polymeneas and Meliopoulos (2016) by using an ellipsoid in the active/reactive power domain to approximate their aggregated flexibility.

Since the flexibility services can be also provided by controllable DRES, Bucher et al., (2015) defines the concept of locational flexibility and uses a robust procurement algorithm to ensure that it can deal with the corresponding forecast uncertainty. Within the same subject, Zhao et al., (2016) details a flexibility metric to assess the largest variation range of uncertainty that the system can support while keeping the system security.

The current regulatory frameworks assign all the responsibility in ensuring the system security to the transmission system operator (TSO). However, the flexible DER and controllable DRES that can help the TSO to accomplish this task are mainly located in the distribution system. This underlines the importance of increasing the coordination between the TSO and the distribution system operator (DSO). For instance, the FP7 EU project evolvDSO project (Six et al., 2014) defined a set of evolving and new DSO roles, which covered the importance of having the DSO contributing to the overall system security. The current interaction processes between TSO and DSO are reviewed in Zegers and Brunner (2014). To do so, six specified grid operation challenges are analyzed, and the conclusion is that a bidirectional data exchange process between TSO/DSO and DSO/Flexible Resources needs to be implemented. Recent works showed the added value of estimating the feasible nodal power injections for DSO, allowing them to determine acceptable levels of flexibility activation given a set of performance requirements. In this scope, the framework proposed in Krause et al., (2009) focus on trace how the operational constraints, whose boundaries are described by convex sets, are mapped to the nodal power complex domain. Given the fact that operational constraints can be modeled by convex polytopes, in Al-digs and Chen (2016) one finds a feasible set of active power nodal injections near the current operating point, by propagating a specific constraint polytope through the solution of a linearly constrained least-square optimization problem. Conversely, the framework proposed in this paper aggregates the distribution network flexibility in order to estimate the decision margins for changing the primary substation operating point. The work presented in Mathieu et al., (2016) details an interaction model between TSO and DSO that aims to avoid that both system operators simultaneously activate flexibility services with opposite consequences.

Considering what is stated above, the literature review regarding the impact of flexibility services on the TSO-DSO cooperation outlines the need of frameworks to exchange relevant data in order to properly deal with the problems brought out by the increasing DRES penetration. This problem can be addressed by controlling the active and/or reactive power flow at the interface between transmission and distribution systems. Within this context, the concept of technical virtual power plant is presented in Pudjianto et al., (2007) and assumes that a fixed active and reactive power profile in the interface can be ensured by the DSO. The exploitation of market-based flexibility services can help to control these profiles and, therefore, is important to include their impact in these type of studies.
This paper proposes an optimization framework based on solving multiple optimization problems that gives to both TSO and DSO a flexibility area with the information concerning the technically feasible range of active and reactive power in a primary substation for a specific time interval and that results from the activation of flexible DER considering the network technical constraints. A first attempt to provide the available flexibility range is detailed in Heleno et al., (2015) and uses a random sampling (RS) approach to estimate it. The nature of sampling processes using uniform distributions leads to significant limitations in finding all the possible solutions to move the scheduled operating point. The novelty of the algorithm presented in this paper is directly related with the capability of finding the points of the flexibility area perimeter by only updating two coefficients of the optimization problem objective function.

In addition to this introductory Section, the remaining of the paper is organized as follows. Section 2 details the reasons why the pathway to accomplish the desired goal are based on solving optimization problems and presents the corresponding mathematical description. Section 3 details the steps to estimate the flexibility area that are the core of the developed algorithm. In Section 4, two case studies are used to evaluate the methodology effectiveness and a critical analysis is performed. Finally, Section 5 resumes the main conclusions and defines future research directions.

\section{The pathway towards an optimization framework}

The estimation of the flexibility range at the TSO/DSO boundary nodes using the RS approach (Heleno et al., 2015) is based on the simulation of a pre-defined number of power flows (i.e. number of samples) in the distribution network in which the flexibility provided by each resource is randomly selected from the respective flexibility band. One limitation of this approach is due to the fact that there is no certainty regarding the feasibility of each sample solution since it is only evaluated after running the power flow. Therefore, and in order to capture a complete view of the impact that the distribution network flexibility can have on the TSODSO interface power flow, a high computational effort is required. Moreover, this RS approach is characterized by a weak capability of capturing the extreme values of power injection. Although the flexibility provided by each resource is independently sampled through uniform distributions, the injected power in the TSO-DSO interface is no longer uniform.

The work described in Heleno et al., (2015) suggested the development of an optimization model capable to provide the available flexibility area in which the scheduled operating point can be moved while ensuring that the technical network limits are respected.

\subsection{Motivation for adopting an optimization model}

Our approach for estimating the flexibility range at the TSODSO interface passes through the formulation of an optimization problem. The existing interdependency between active and reactive power and the grid technical constraints impact on the amount of flexibility that can be activated in the distribution network thus influencing the available possibilities to move the scheduled operating point. Without this influence, it would become easier to estimate the flexibility range since it would directly result from adding the flexibility provided by each resource to the scheduled operating point. This would result in a rectangle flexibility area. However, the inclusion of the grid technical limits constrain the flexibility area leading to a smaller rectangle, as shown Fig. 1.

Additionally, the PQ interdependency results in a flexibility area with a different shape like the one presented in Fig. 2. Therefore, 


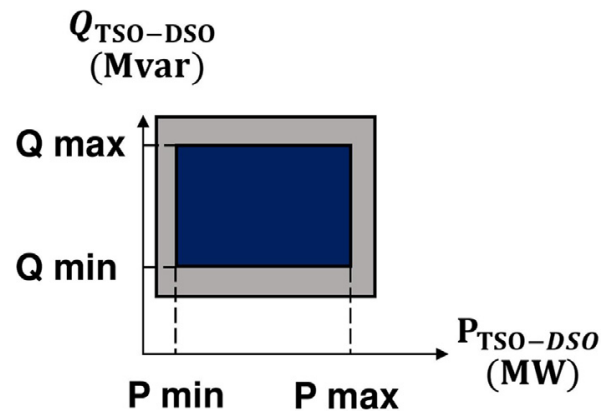

Fig. 1. Flexibility area neglecting the $P Q$ interdependency and the technical grid limitations (grey) and neglecting only the PQ interdependency (blue). (For interpretation of the references to color in this figure legend, the reader is referred to the web version of this article.)

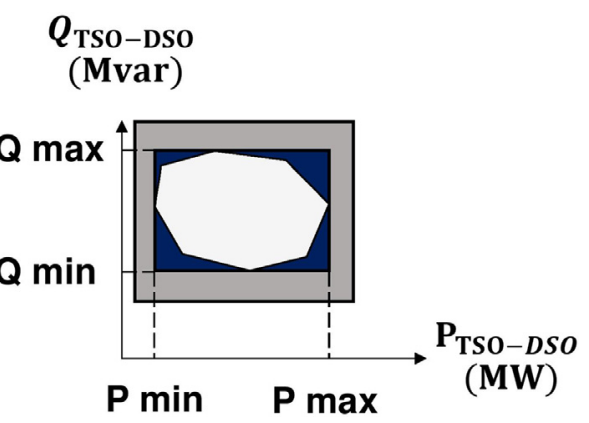

Fig. 2. Flexibility area delimited by the technical grid constraints and considering the PQ interdependency.

it is necessary to find out which is the feasible set of operating points within the smaller rectangle of Fig. 1.

Fig. 2 illustrates in a clear way that a successful method to estimate the flexibility ranges is the one capable to catch the values of power injection that compose the flexibility area perimeter.

\subsection{The optimization problem formulation}

From what was discussed in the previous Section, as a first step the methodology should aim to find the maximum and minimum of active and reactive power flexibility that can be activated in the distribution network. Therefore, it is necessary to solve, at this first stage, four different optimization problems. The novelty behind the proposed methodology is based on developing an adaptive objective function capable to catch all these points. In other words, the goal is to construct one single optimization problem that will be run several times (only changing some parameters of the objective function) in order to allow to find the extreme points of power injection.

The following objective function, with two parameters, is the core of this approach:

$\min \alpha P_{D S O \rightarrow T S O}+\beta Q_{D S O \rightarrow T S O}$

where $P_{D S O \rightarrow T S O}$ and $Q_{D S O \rightarrow T S O}$ are the active and reactive power flows at the TSO-DSO interface. $\alpha$ and $\beta$ are the two coefficients that are updated during the optimization procedure thus allowing to completely explore the flexibility area perimeter. In fact, this objective function illustrates a family of straight lines whose slope $\theta$ can be obtained from $\tan \theta=-\alpha \mid \beta$. Therefore, by minimizing the objective function for different slopes, the optimization problem makes use of the tangent lines of the flexibility area perimeter to intersect the $P_{D S O \rightarrow T S O}$ and $Q_{D S O \rightarrow T S O}$ plan. In fact, as detailed in Section 3, the proposed method iteratively maximizes or minimizes the objective function due to the different values of the co- efficients $\boldsymbol{\alpha}$ and $\boldsymbol{\beta}$ thus allowing to explore the upper and lower zones of the flexibility perimeter.

The decision variables that characterize this optimization problem are linked with the flexible resources. Therefore, the active and reactive power flexibility activated in each distributed generator (DG), load, reactive power compensator or on-load tap changer (OLTC) are described by:

$$
\begin{aligned}
& \Delta P_{h}^{D G}, \Delta Q_{h}^{D G} \forall h \in N_{G} \\
& \Delta P_{i}^{\text {Demand }}, \Delta Q_{i}^{\text {Demand }} \forall i \in N_{L} \\
& \Delta Q_{c}^{\text {cond }} \forall c \in N_{c} \\
& \Delta \text { OLTC }_{i j}^{t} \forall t \in T
\end{aligned}
$$

where $\boldsymbol{h}$ represents each DG, $\boldsymbol{i}$ each load, and $\boldsymbol{c}$ each reactive power compensator of the corresponding sets $\left(\boldsymbol{N}_{\boldsymbol{G}}, \boldsymbol{N}_{\boldsymbol{L}}\right.$ and $\left.\boldsymbol{N}_{\boldsymbol{c}}\right)$. Concerning the flexibility provided the OLTC, it is dependent of the number of tap positions. $\boldsymbol{t}$ is linked with each specific OLTC of the $\boldsymbol{T}$ set. Lastly, the voltage magnitude in the bus reference is also considered as a decision variable of the optimization problem.

The constraints to which this optimization problem is subjected are the same of a typical optimal power flow study (OPF) (Carpentier, 1992). The formal and complete formulation of the optimization problem is presented in Appendix B. The proposed methodology solves several optimization problems that are characterized by a highly non-linear behavior. Several methods to solve the OPF problem are available and studied in the literature. The Newton's method (Monticelli and Liu, 1992), the linear programming method (Stott et al., 1979) and the quadratic programming method (Burchett et al., 1984) are examples. Standard integer programming methods as branch and bound algorithm (Haffner et al., 2001; Hogan et al., 2005) are non-polynomial which explains the high computational effort when dealing with large-scale problems. On the other hand, rounding-off based method (Tinney et al., 1988; Liu et al., 1992) does not require a high computational time, but shows problems in find optimality. The interior point methods have been extensively used to solve OPF problems mainly for two reasons: fast convergence and effectiveness when dealing with inequalities. These methods can be sub-divided in three different categories: projective (Karmarkar, 1984; Gill et al., 1986), affine-scaling (Marsten et al., 1989; Saigal, 1995) and the primaldual methods (Gonzaga, 1992; Megiddo, 1989). This last class of interior-point methods serve as basis to solve the OPF problems that the proposed methodology faces since it shows a high performance both in terms of computational time and optimality. Instead of focusing on detailing the interior point-method used (which is properly detailed in the references), the following Section presents step-by-step the optimization procedure that allows to draw the flexibility area.

\section{Proposed optimization procedure}

The order by which the several optimization problems are solved, the update of $\alpha$ and $\beta$ coefficients and the convergence criterion are detailed in this Section.

Fig. 3 sums up the main steps of the optimization procedure that leads to the flexibility area estimation. In a more detailed way, this procedure can be described by the following phases:

1. Optimize for $\theta=0$ o and for $\theta=180^{\circ}$ in order to obtain the extreme values of reactive power injection $Q_{\min }$ and $Q_{\max }$ and their corresponding active power values at the TSO-DSO interface. Therefore, $\beta=0$ and $\alpha= \pm 1$.

2. Optimize for $\theta= \pm 90$ 。 in order to obtain the extreme values of active power injection $P_{\min }$ and $P_{\max }$ and their corresponding reactive power values at the TSO-DSO interface. Therefore, $\beta= \pm 1$ and $\alpha=0$. 


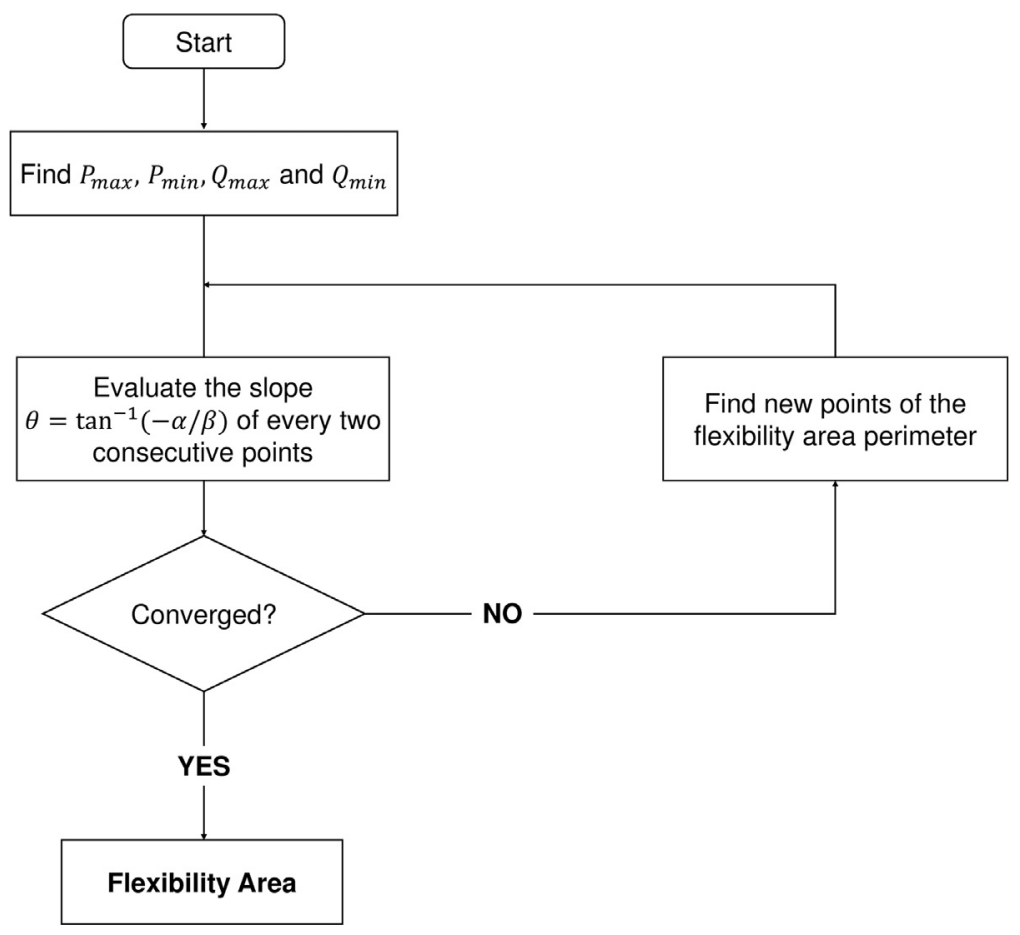

Fig. 3. Optimization procedure step-by-step.

These two first steps allow to have a first idea of the flexibility area shape. In order to enhance the representation of this area, more points on the flexibility area perimeter should be estimated. The following step belongs to a closed loop that is dependent of the defined convergence criterion.

3. For each two consecutive points on the area perimeter, calculate the angle $\theta_{\text {new }}=\tan ^{-1}\left(\frac{Q_{i}-Q_{i+1}}{P_{i}-P_{i+1}}\right)$ of the straight line joining them and then compare it with the angles that have generated those two points. If the two following inequalities are respected, optimize for $\theta_{\text {new }}$ in order to find a new point between the two original ones.

$\left|\theta_{\text {new }}-\theta_{i}\right|>\theta_{\text {tol }}$

$\left|\theta_{\text {new }}-\theta_{i+1}\right|>\theta_{\text {tol }}$

where $\theta_{\text {tol }}$ is the tolerance angle.

The process stops when there is no more $\theta_{\text {new }}$ respecting these two inequalities.

Fig. 4 is a simple example of the process carried on. In this case, three extreme points were already obtained through the corresponding tangent straight lines (solid lines $-\theta=0,90,180$ o). Let us now consider that the convergence criterion is not respected regarding both pairs of consecutive points. Therefore, the slope of the straight line joining these points (dashed lines) provides the new tangent lines (dotted lines) that intersect the PQ perimeter. This means that two new optimization problems for the correspondent $\theta_{\text {new }}$ need to be solved. Thus, the proposed method, by finding the intersection between these two new tangent lines (dotted lines) and the perimeter of the flexibility region, provides two new operating points lying on it.

The closed loop comes to an end when all the new angles $\theta_{\text {new }}$ would lead to points on the flexibility area perimeter that would not have a considerable contribution to the variation of its shape.

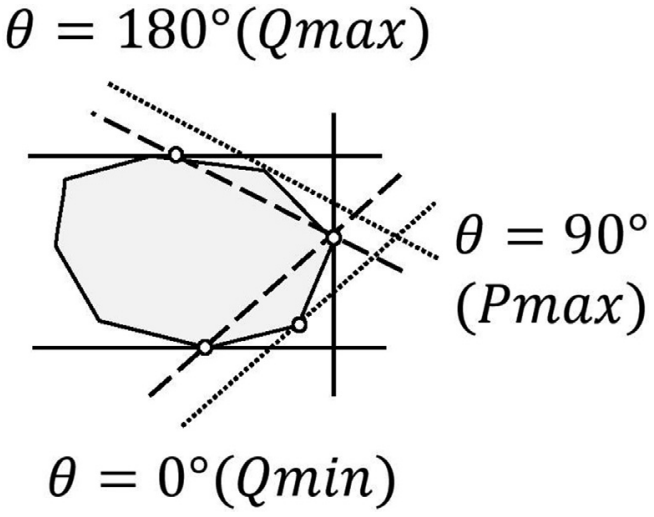

Fig. 4. Methodology illustration base on the tangent lines.

Thus, the convergence criterion is based on the comparison between the $\theta_{\text {new }}$ and the angles that led to the appearance of the consecutive points $i$ and $i+1$ (i.e. $\theta_{i}$ and $\theta_{i+1}$ ).

This optimization model is based on solving a set of optimization problems that return the operating points belonging to the limit of the flexibility area. The convergence procedure, by controlling the slopes, avoids to perform unnecessary optimizations for $\theta_{\text {new }}$ that would not bring significant changes for the current shape of the flexibility area.

\section{Case studies}

\subsection{Test networks description}

The algorithm effectiveness was assessed using a modified version of the IEEE 9 bus test system whose characteristic are reported in Appendix A. This test network has a 250MVA transformer with fixed tap position connected to a primary substation. The three loads connected to system represent an aggregated consumption of down level networks of 315 MW and 115 Mvar of 


\section{Flexibility area}

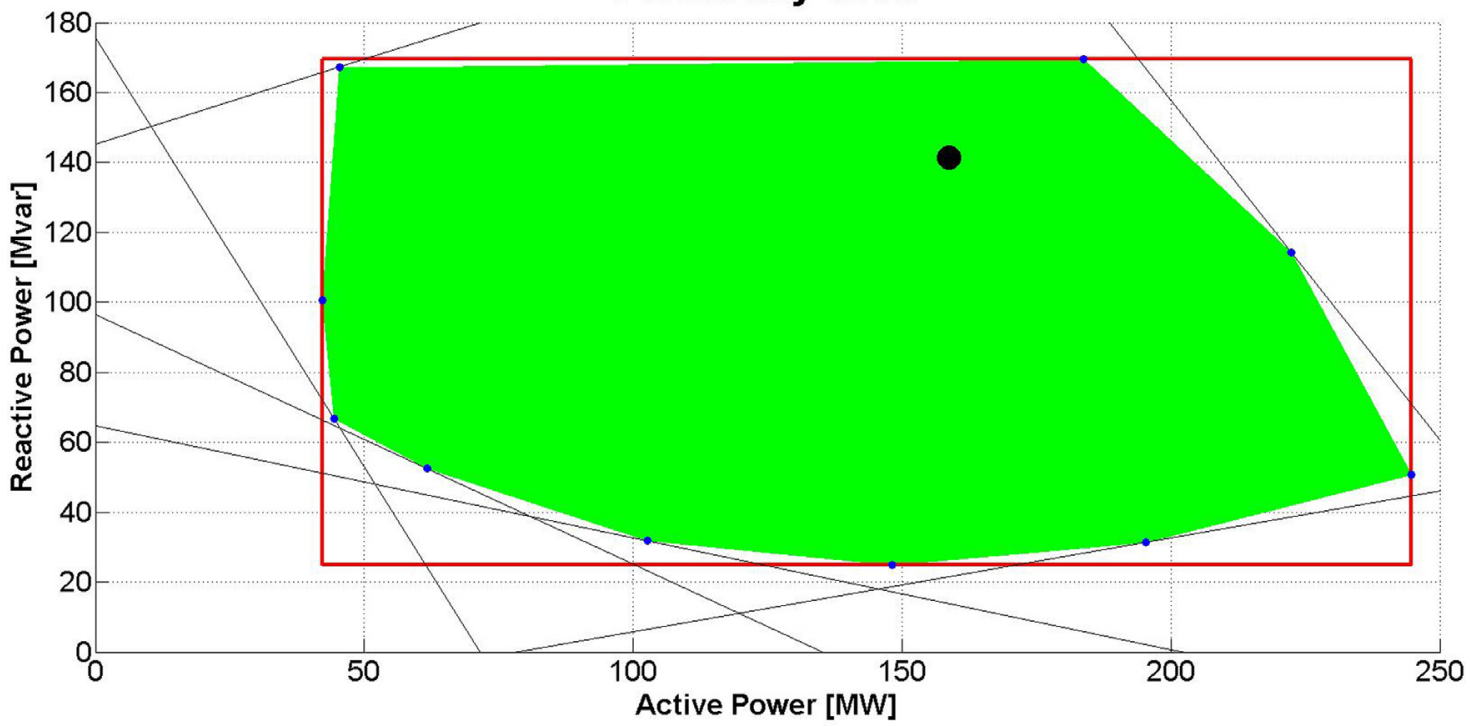

Fig. 5. Flexibility area for the IEEE 9 bus test system $\left(\theta_{\text {tol }}=10^{\circ}\right)$. (For interpretation of the references to color in this figure, the reader is referred to the web version of this article.)

Table 1

Degrees of flexibility for each flexible resource.

\begin{tabular}{lll}
\hline Resource & \multicolumn{2}{l}{ Flexibility degree } \\
\hline Synchronous compensators & $Q_{\text {injection }}$ & $100 \%$ installed capacity \\
& $P_{\text {curtailment }}$ & - \\
DRES & $Q_{\text {injection }}$ & $6,67 \%$ installed capacity \\
& $P_{\text {curtailment }}$ & $100 \%$ available power \\
Interruptible consumers & $Q_{\text {curtailment }}$ & $10 \%$ reactive load \\
& $P_{\text {curtailment }}$ & $10 \%$ active load \\
\hline
\end{tabular}

total active and reactive power. Concerning the flexible resources there are one synchronous compensator (1 unit of 150 Mvar), one wind park with $300 \mathrm{MW}$ of installed capacity and interruptible consumers.

The network above described has the proper characteristics to present an illustrative example of the methodology merits. However, in order to show the algorithm applicability to larger distribution networks, a test was performed in a modified version of the 210 bus test system (Madureira, 2010). Five interruptible consumers, nine DRES (totalizing 5.1 MW of installed capacity) and one synchronous compensator (1 unit of 1 Mvar) compose the available flexible resources. Moreover, a 10 MVA transformer with fixed tap position is connected within the network.

Table 1 shows the flexibilities that would be activated in the networks if the technical constraints enable. In another test stage, the available transformers have OLTC capability and the synchronous compensators have been substituted by capacitor banks thus allowing to observe the impact of discrete variables upon the flexibility area.

\subsection{Results}

Fig. 5 shows the flexibility area obtained for the IEEE 9 bus test system described in Section 4.1. More precisely, it shows the area in which the scheduled operating point (black point) can be moved due to the activation of flexible resources without any violation of the network technical limits.

As shown in Fig. 5, the flexibility area has both active and reactive power ranges which is in accordance with the expectations due to the available flexible resources. In fact, there is more flexibility available in the network, but the technical limits constraint its activation. For instance, in Fig. 5, it is clearly observable that the active power upper limit is constrained by the transformer rated power.

The red rectangle that connects the extreme points of the perimeter is the illustration of which would be the flexibility area if the PQ interdependency would not impact on the maximum and minimum values of active and reactive power, as it was already illustrated in Fig. 1 by the smaller rectangle. Since this impact affects the flexibility area shape, the methodology searches for other points on the perimeter which are obtained using the tangent straight lines shown in Fig. 5 (i.e. blue points). In this case, a $\theta_{\text {tol }}=10^{\circ}$ tolerance has been chosen thus allowing to obtain a representative idea of the flexibility area shape.

Fig. 6 presents the flexibility area obtained for the same network that led to the one of Fig. 5 however using a $\theta_{\text {tol }}=1^{\circ}$ of tolerance. This means that the procedure continues to explore the space between two consecutive points even with a small difference among the angles from which they have been generated. By comparing Figs. 5 and 6, it is clear the importance of define a tradeoff between the area representation and the computational effort. A lower tolerance angle leads to a clear increase of the points obtained by the optimization procedure and therefore of the computational time required, while the variations of the flexibility area shape could be not significant.

Regarding the flexibility area obtained for the larger network, the conclusions to be extracted are similar.

As expected, Fig. 7 shows a flexibility area with active and reactive power flexibility ranges which are clearly constrained by the network limits and the PQ interdependency. Comparing with the results obtained for the IEEE 9 bus test system, the active power upper limit is also constrained, but in this case by the branch that connects the primary substation to the distribution network (2 MVA of maximum branch flow capacity).

The optimization model fulfills the expectations by providing the aggregated active and reactive power flexibility available at the TSO-DSO boundary nodes. The update of the coefficients $\alpha$ and $\beta$ proved to be an effective procedure to obtain the tangent straight lines that, by intersecting the PQ plan, provide new feasible points on the flexibility area perimeter.

The effectiveness of this methodology was compared with the RS approach presented in Heleno et al. (2015) by assessing the per- 


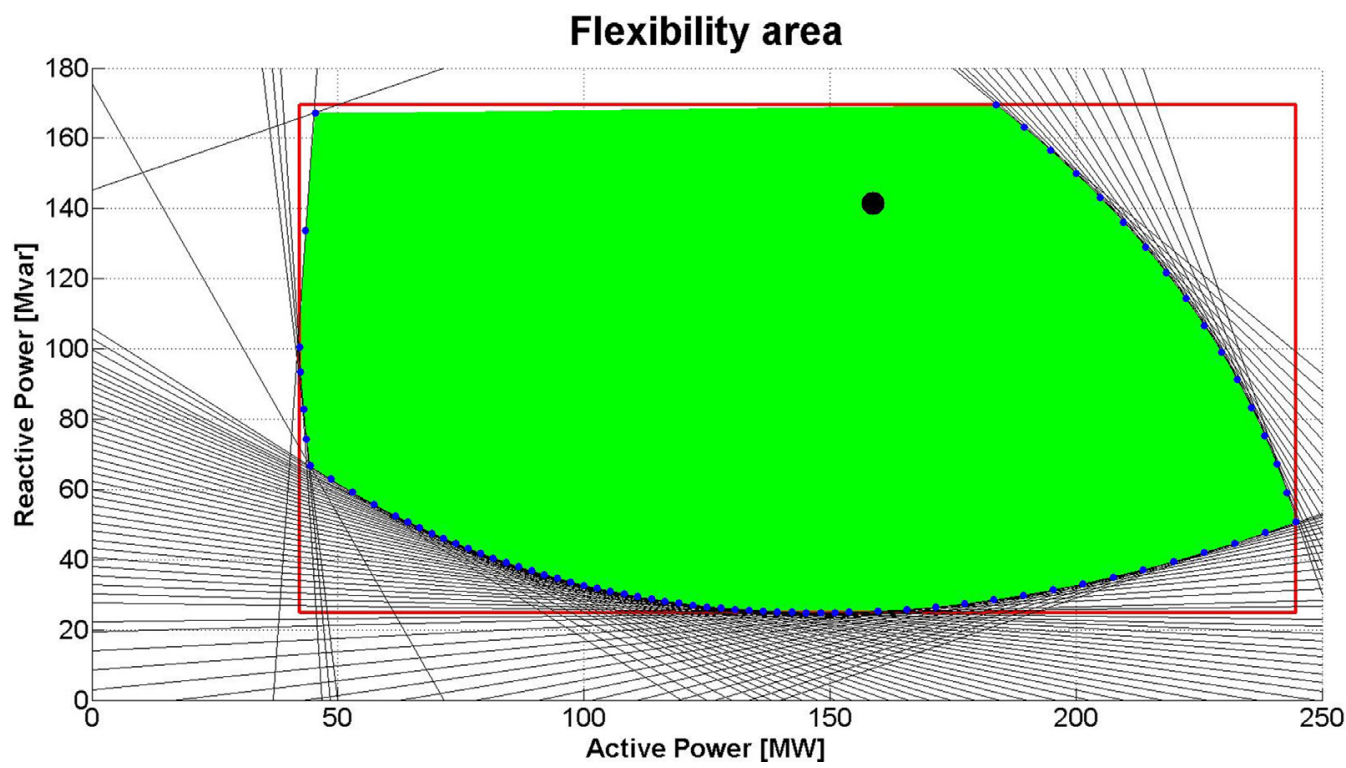

Fig. 6. Flexibility area for the IEEE 9 bus test system $\left(\theta_{t o l}=1^{\circ}\right)$.

Flexibility area

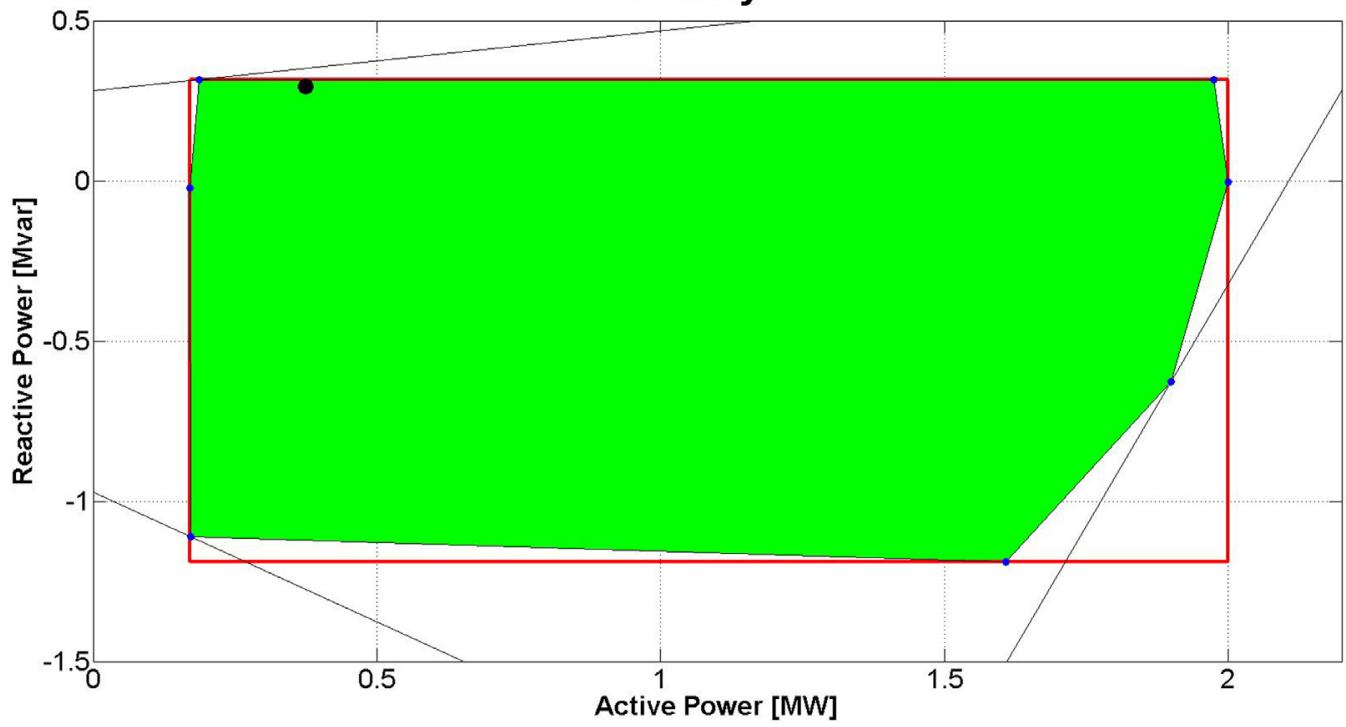

Fig. 7. Flexibility area for the 210 bus system $\left(\theta_{\text {tol }}=10^{\circ}\right)$.

formance achieved in terms of increase of the flexibility area identified and reduction of computational effort:

Area Increase $=\frac{A_{\text {Opt_model }}-A_{R S}}{A_{R S}} \times 100 \%$

Computation time reduction $=\frac{T_{R S}-T_{\text {Opt_model }}}{T_{R S}} \times 100 \%$

where $A_{\text {Opt_model }}, T_{\text {Opt model }}, A_{R S}$ and $T_{R S}$ are the flexibility areas and computational times obtained with the methodology presented in this paper and the RS approach.

The comparison has been performed for both test networks, considering a convergence tolerance of $10^{\circ}$ and by running the RS for 1000, 10,000 and 100,000 samples.

Each red point in Figs. 8 and 9 illustrates a feasible result of a power flow in the distribution networks. Assuming that the perimeter of the flexibility area is convex, the convex hull of these points gives the variation margin of the operating point obtained with the RS approach which are clearly lower than the one obtained by the proposed methodology.
Table 2 shows that the proposed algorithm allows to achieve a clear increase in the identification of the flexibility area within less computational time, which was expected due to the limitations found in the RS. Thus, the methodology catches the full contribution of each flexibility resource available thus providing to both TSO and DSO a complete idea of which changes are feasible in the scheduled operating point. The actual solution times of the developed approach and the random sampling are presented are presented in Table 3.

Considering these running times, the applicability of the proposed method to real-life situations (e.g. hourly forecasts, changes on the network topology) do not present any barrier regarding the time constraints that can be imposed by the network operation. As remark, MIPS (Matpower Interior Point Solver) (Zimmerman et al., 2011) was the chosen solver to run the OPFs and a computer with the following characteristics was used: OS: Windows 7 Enterprise (64 bit), 8 GB of memory, Intel® Core ${ }^{\mathrm{TM}} \mathrm{i} 7-2600 \mathrm{CPU} @ 3.40 \mathrm{GHz}$.

The following test stage includes the appearance of discrete variables in the optimization problems corresponding to the pres- 


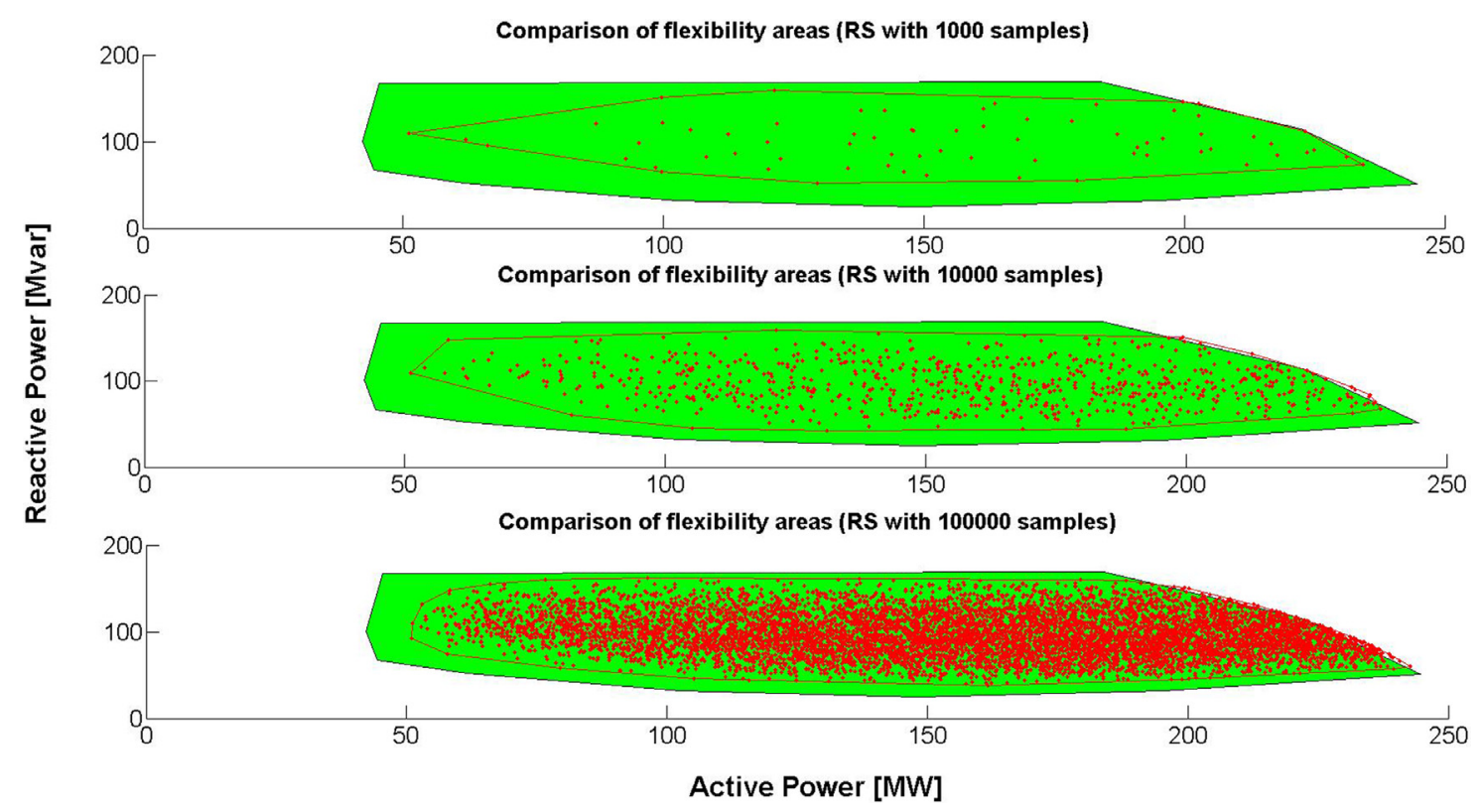

Fig. 8. Comparison of the flexibility areas provided by the algorithm and the RS approach (IEEE 9 bus test system).

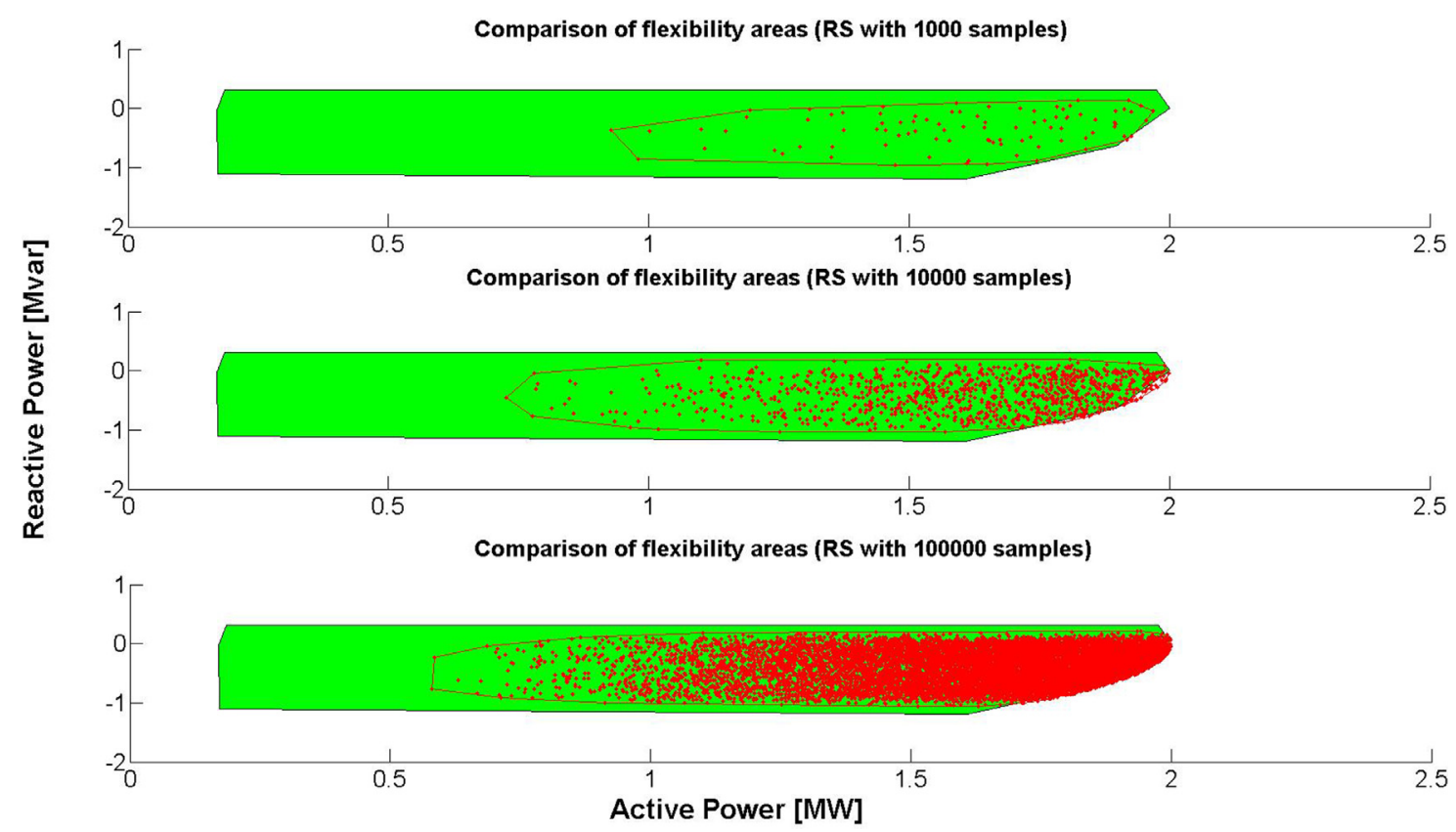

Fig. 9. Comparison of the flexibility areas provided by the algorithm and the RS approach (210 bus system).

Table 2

Comparison with the RS approach.

\begin{tabular}{llllllll}
\hline \multirow{2}{*}{ Network/No. samples } & \multicolumn{3}{c}{ Flexibility area increase (\%) } & & \multicolumn{2}{c}{ Computational time reduction (\%) } \\
\cline { 2 - 3 } & 1000 & 10000 & 100000 & & 1000 & 10000 & 100000 \\
\hline IEEE 9 bus test system & 72.67 & 37.93 & 24.10 & & 57.55 & 96.08 & 99.56 \\
210 bus test system & 187.69 & 92.35 & 64.28 & & 51.83 & 95.39 & 99.49 \\
\hline
\end{tabular}

ence of OLTCs with 3 tap positions (0.95 1.00 and 1.05) in "per unit" system and capacitor banks with 50\% (IEEE 9 bus test system) and $100 \%$ (210 bus test system) of step length. The aim of these tests is only to understand if the inclusion of a discrete nature leads also to a discrete solution thus making it impossible to describe the flexibility area by the envelope of the feasible points. In other words, Section 4.3 does not intend to test the developed algorithm when considering discrete variables. The effectiveness of the proposed approach is only assessed and proved when dealing with continuous flexible resources since this leads to convex flexibility regions.

\subsection{Discussion}

In order to understand the impact of discrete variables on the flexibility area, the optimization procedure described in Section 

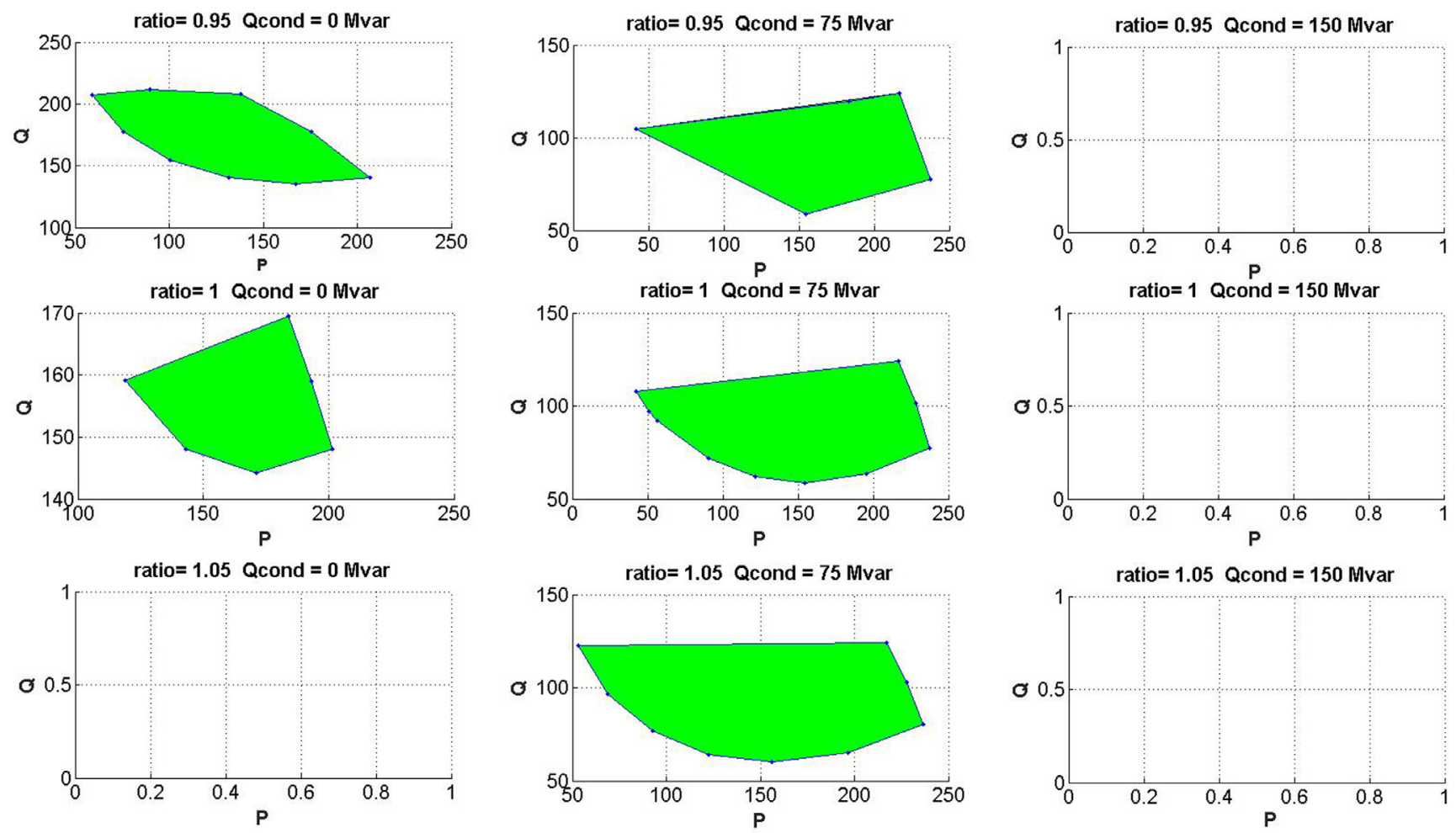

Fig. 10. Flexibility areas for each different combination of discrete variables (IEEE 9 bus test system).

Table 3

Computational time of both approaches.

\begin{tabular}{lllll}
\hline \multirow{2}{*}{ Network/No. samples } & \multicolumn{4}{l}{ Computational time $(\mathrm{s})$} \\
\cline { 2 - 5 } & 1000 & 10000 & 100000 & Proposed method \\
\hline IEEE 9 bus test system & 1.67 & 19.9 & 163.32 & 0.88 \\
210 bus test system & 5.71 & 59.66 & 533.6 & 2.75 \\
\hline
\end{tabular}

3 has been run for each of the 9 and 6 different combinations of discrete values with a tolerance parameter of $10^{\circ}$. This enumeration should be seen as a trial to clearly show how discrete variables influence the flexibility area and not as practical procedure to estimate it. As matter of fact, the enumeration of all the combinations is possible only if a limited number of tap and capacitor bank positions is considered, otherwise it would increase dramatically the computational effort.

Fig. 10 shows that within the 9 available discrete variables combinations, only 5 lead to the feasible areas. In the other 4 combi-

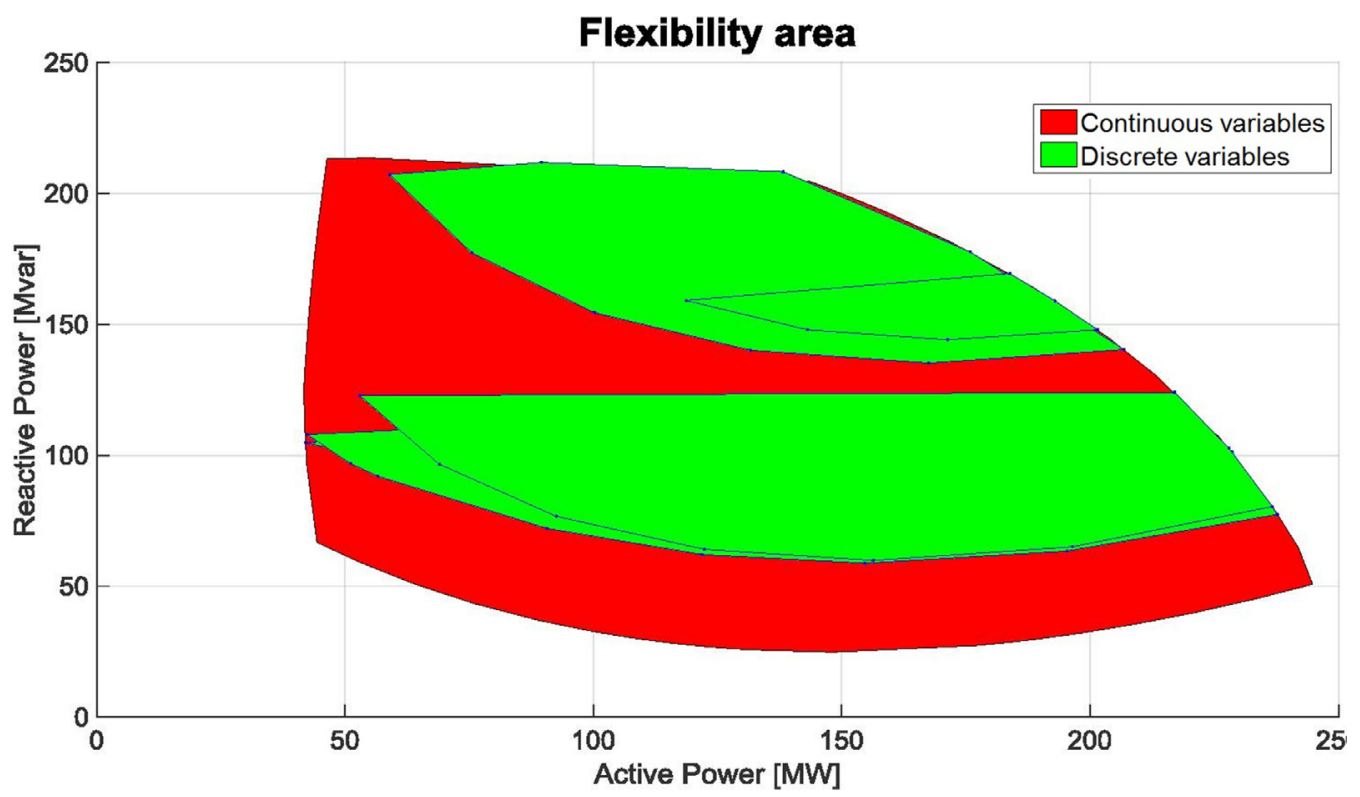

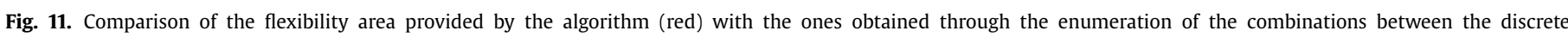

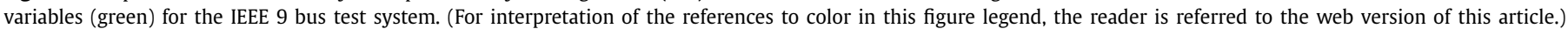




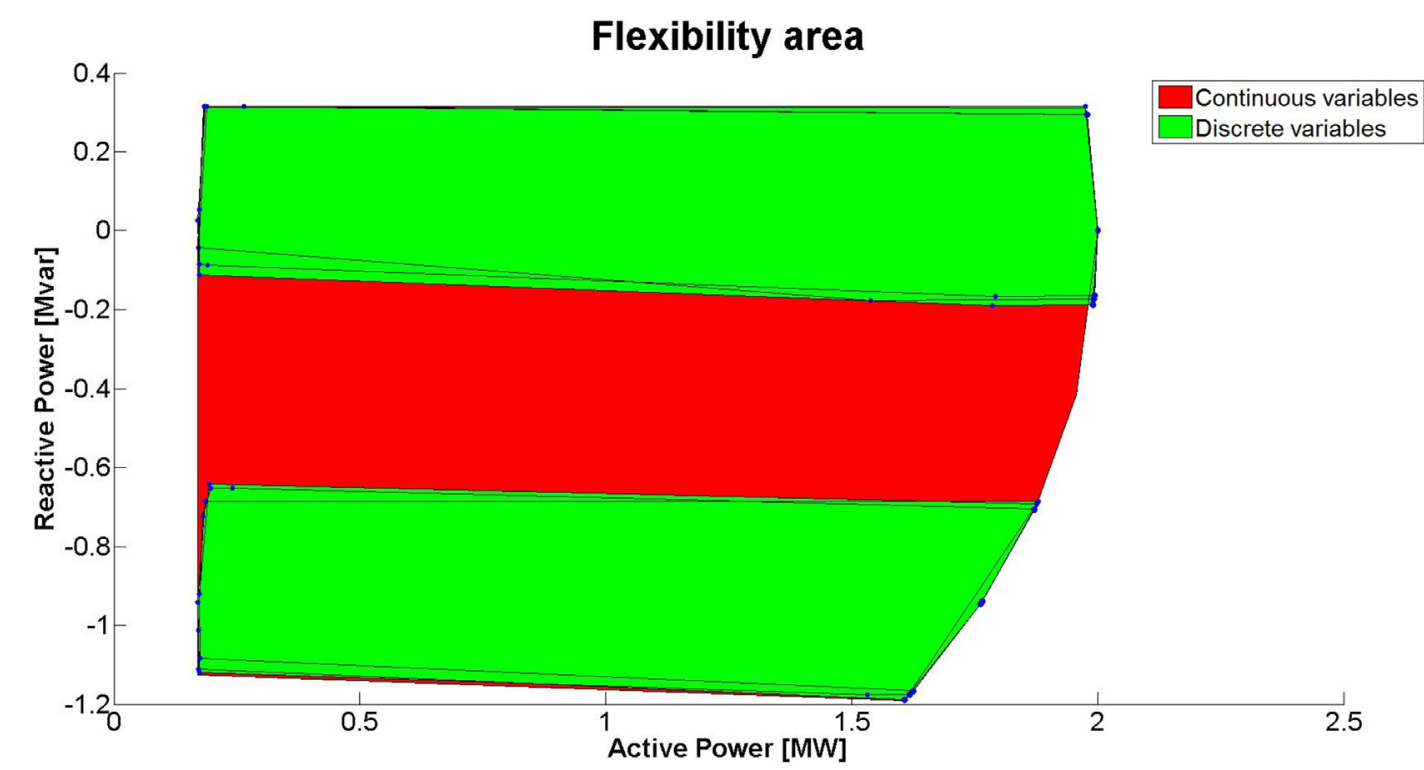

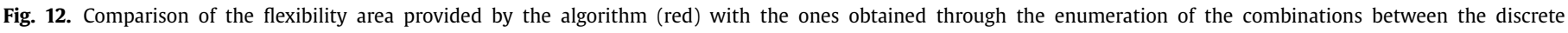
variables (green) for 210 bus test system. (For interpretation of the references to color in this figure legend, the reader is referred to the web version of this article.)

nations, no feasible areas are found since there is no solution for the optimization problems in the specific conditions described in Fig. 10. The flexibility domain is composed by the union of the feasible sub-sets (green areas), as shown in Fig. 11.

The red area represents the margin of variation of the operating point when the discrete variables are replaced by continuous variables, within the corresponding ranges (relaxation of integrality constraints). The same comparison was performed for the 210 bus test system, as shown in Fig. 12.

By considering all the variables as continuous, it becomes clear that simple optimizations, finding vertex points, would lead to an over-estimation of the size of the flexibility area. In fact, the red area is larger than the convex hull enclosing the green disjoint flexibility areas. Even a method capable to obtain this convex hull would represent a domain notoriously larger than the feasible domain - and this is a serious warning against optimistic but unrealistic estimations, based on simple approximations and relaxation of variables.

\section{Conclusions}

This work is a contribution in the development of decision-aid tools that permit to increase the bidirectional exchange of information between TSO and DSO in order to keep the system security while increasing the hosting capacity of DRES in distribution systems. The proof of concept was obtained by using two test networks and comparing the results with a random sampling approach. A significant increase of the flexibility area with respect to the random sampling approach was achieved, thus proving the merit of the proposed optimization model in providing more information to the system operators in terms of decision margin for changing the primary substation operating point. Furthermore, it reduces the computational effort of the random sampling approach.

The developed optimization model proved its effectiveness when dealing only with continuous variables since the corresponding flexibility area is also continuous. However, when considering discrete variables, it is neither possible to guarantee that the flexibility domain remains convexly connected, nor to assure that it remains connected, as it may become composed of disjoint parts. In this context, an inspection of the entire search space defined by the integer variables is crucial to help in the identification of the unfeasible regions within the flexibility area. The technique described in this paper allows for such inspection, in an enumeration fashion, which has limitations when the number of variables grows beyond a reasonable limit.

The standard method proposed in the paper reaches a definition of the convex hull of the flexibility region. This has the potential to become a practical method, used as a guidance or decisionaid tool, suggesting alternative operating points. However, the paper shows that, at present, such suggestions should be verified for feasibility, because of the effects resulting from the discrete features of the real problem.

This tool is likely to be very useful for DSOs, to define active and reactive power interchanges limits with the transmission system, while avoiding penalties for exceeding operation limits - and for TSOs, because it avoids them the definition of operation conditions in the interconnection that are actually unfeasible and cannot be met by the distribution systems.

\section{Acknowledgments}

The research leading to this work has been carried out as a part of the evolvDSO project (Development of methodologies and tools for new and evolving DSO roles for efficient DRES integration in distribution networks). This project is funded by the European Commission under the seventh framework program (FP7) under grant agreement number 608732 .

This work was also supported in part by the Portuguese Foundation for Science and Technology (FCT) under the grant SFRH/BD/124727/2016.

\section{Appendix A}

Tables A.1, A.2, A.3.

\section{Appendix B}

This appendix intends to provide a formal and complete formulation of the optimization problem described in this manuscript.

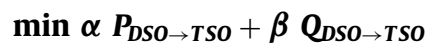


Table A.1

Nodes and load data.

\begin{tabular}{llll}
\hline $\begin{array}{l}\text { Node } \\
\text { number }\end{array}$ & $\begin{array}{l}\text { Voltage } \\
\text { level }(\mathrm{kV})\end{array}$ & $\begin{array}{l}\text { Active Power } \\
\text { Load (MW) }\end{array}$ & $\begin{array}{l}\text { Reactive Power } \\
\text { Load (Mvar) }\end{array}$ \\
\hline 1 & 150 & 0 & 0 \\
2 & 20 & 0 & 0 \\
3 & 20 & 0 & 0 \\
4 & 60 & 0 & 0 \\
5 & 60 & 90 & 30 \\
6 & 60 & 0 & 0 \\
7 & 60 & 100 & 35 \\
8 & 60 & 0 & 0 \\
9 & 60 & 125 & 50 \\
\hline
\end{tabular}

Table A.2

Branch data.

\begin{tabular}{llllll}
\hline Node $i$ & Node $j$ & $\mathrm{R}(\mathrm{p} . \mathrm{u})$ & $\mathrm{X}(\mathrm{p} . \mathrm{u})$ & $\mathrm{B}(\mathrm{p} . \mathrm{u})$ & $\begin{array}{l}\text { Maximum Flow } \\
\text { Capacity (MVA) }\end{array}$ \\
\hline 4 & 5 & 0.017 & 0.092 & 0 & 250 \\
5 & 6 & 0.039 & 0.17 & 0 & 150 \\
3 & 6 & 0 & 0.0586 & 0 & 300 \\
6 & 7 & 0.0119 & 0.1008 & 0 & 150 \\
7 & 8 & 0.0085 & 0.072 & 0 & 250 \\
8 & 2 & 0 & 0.0625 & 0 & 250 \\
8 & 9 & 0.032 & 0.161 & 0 & 250 \\
9 & 4 & 0.01 & 0.085 & 0 & 250 \\
1 & 4 & 0 & 0.0576 & 0 & 250 \\
\hline
\end{tabular}

Table A.3

Generator data.

\begin{tabular}{llll}
\hline $\begin{array}{l}\text { Node } \\
\text { number }\end{array}$ & Type & $\begin{array}{l}\text { Active power } \\
\text { range }\end{array}$ & $\begin{array}{l}\text { Reactive power } \\
\text { range }\end{array}$ \\
\hline 2 & Wind Park & $0-300$ & $0-20$ \\
3 & Synchronous Compensator & 0 & $0-150$ \\
\hline
\end{tabular}

Subjected to:

$\left(\Delta \boldsymbol{P}_{n}^{D G}+\boldsymbol{P}_{n}^{D G}\right)-\left(\Delta \boldsymbol{P}_{n}^{\text {Demand }}+\boldsymbol{P}_{n}^{\text {Demand }}\right)-\boldsymbol{P}_{n}=0, \quad \forall \boldsymbol{n} \in \boldsymbol{N}$

$$
\begin{aligned}
& \left(\Delta \boldsymbol{Q}_{n}^{D G}+\boldsymbol{Q}_{n}^{D G}\right)+\left(\Delta \boldsymbol{Q}_{n}^{\text {cond }}+\boldsymbol{Q}_{n}^{\text {cond }}\right)-\left(\Delta \boldsymbol{Q}_{n}^{\text {Demand }}+\boldsymbol{Q}_{n}^{\text {Demand }}\right) \\
& -\boldsymbol{Q}_{n}=0, \quad \forall n \in N
\end{aligned}
$$

$$
\boldsymbol{V}_{\boldsymbol{n}, \boldsymbol{m i n}} \leq\left|\boldsymbol{V}_{\boldsymbol{n}}\right| \leq \boldsymbol{V}_{\boldsymbol{n}, \boldsymbol{m a x}}, \forall \boldsymbol{n} \in \boldsymbol{N}
$$

$\boldsymbol{\theta}_{\text {ref }}=0$

$$
Q_{n}^{\text {cond }} \in\left\{Q_{n}^{\text {cond }}\right\}, \forall n \in N_{c}
$$

$$
\operatorname{OLTC}_{i j}^{t} \in\left\{\operatorname{OLTC}_{i j}^{t}\right\}, \forall t \in T
$$

$\left|\boldsymbol{S}_{\boldsymbol{i j}}^{\boldsymbol{b}}\right|^{2} \leq\left(\boldsymbol{S}_{\max }^{\boldsymbol{b}}\right)^{2}, \forall \boldsymbol{b} \in \boldsymbol{B}$

$\left|\boldsymbol{S}_{\boldsymbol{j i}}^{\boldsymbol{b}}\right|^{2} \leq\left(\boldsymbol{S}_{\max }^{\boldsymbol{b}}\right)^{2}, \forall \boldsymbol{b} \in \boldsymbol{B}$

$$
\Delta \boldsymbol{P}_{h, \text { min }}^{D G} \leq \Delta \boldsymbol{P}_{h}^{D G} \leq \Delta \boldsymbol{P}_{h, \max }^{D G} \forall \boldsymbol{h} \in \boldsymbol{N}_{\boldsymbol{G}}
$$

$\Delta \boldsymbol{Q}_{\boldsymbol{h}, \min }^{D G} \leq \Delta \boldsymbol{Q}_{\boldsymbol{h}}^{D G} \leq \Delta \boldsymbol{Q}_{h, \max }^{D G} \forall \boldsymbol{h} \in \boldsymbol{N}_{\boldsymbol{G}}$

$\Delta \boldsymbol{P}_{i, \min }^{\text {Demand }} \leq \Delta \boldsymbol{P}_{i}^{\text {Demand }} \leq \Delta \boldsymbol{P}_{i, \max }^{\text {Demand }} \forall i \in \boldsymbol{N}_{L}$

$$
\Delta \boldsymbol{Q}_{i, \min }^{\text {Demand }} \leq \Delta \boldsymbol{Q}_{\boldsymbol{i}}^{\text {Demand }} \leq \Delta \boldsymbol{Q}_{\boldsymbol{i}, \max }^{\text {Demand }} \forall \boldsymbol{i} \in \boldsymbol{N}_{L}
$$

$$
\Delta \boldsymbol{Q}_{c, \min }^{\text {cond }} \leq \Delta \boldsymbol{Q}_{c}^{\text {cond }} \leq \Delta \boldsymbol{Q}_{c, \max }^{\text {cond }} \forall \boldsymbol{c} \in \boldsymbol{N}_{\boldsymbol{c}}
$$

where:

$$
\begin{aligned}
& \boldsymbol{P}_{\boldsymbol{n}}=\left|\boldsymbol{V}_{\boldsymbol{n}}\right| \sum_{\boldsymbol{k}=1}^{\boldsymbol{N}}\left[\left|\boldsymbol{V}_{\boldsymbol{k}}\right|\left(\boldsymbol{G}_{\boldsymbol{n} \boldsymbol{k}} * \cos \boldsymbol{\theta}_{\boldsymbol{n} \boldsymbol{k}}+\boldsymbol{B}_{\boldsymbol{n} \boldsymbol{k}} * \sin \boldsymbol{\theta}_{\boldsymbol{n} \boldsymbol{k}}\right)\right] \\
& \boldsymbol{Q}_{\boldsymbol{n}}=\left|\boldsymbol{V}_{\boldsymbol{n}}\right| \sum_{\boldsymbol{k}=1}^{\boldsymbol{N}}\left[\left|\boldsymbol{V}_{\boldsymbol{k}}\right|\left(\boldsymbol{G}_{\boldsymbol{n} \boldsymbol{k}} * \sin \boldsymbol{\theta}_{\boldsymbol{n} \boldsymbol{k}}-\boldsymbol{B}_{\boldsymbol{n} \boldsymbol{k}} * \cos \boldsymbol{\theta}_{\boldsymbol{n} \boldsymbol{k}}\right)\right]
\end{aligned}
$$

$\boldsymbol{N}$ and $\boldsymbol{B}$ illustrate the number of nodes and branches that compose the network. The activated active and reactive power flexibility in each node $\mathrm{n}$ are described by $\Delta \boldsymbol{P}_{n}^{\boldsymbol{D G}}, \Delta \boldsymbol{Q}_{n}^{\boldsymbol{D G}}, \Delta \boldsymbol{P}_{n}^{\text {Demand }}$, $\Delta \boldsymbol{Q}_{n}^{\text {Demand }}, \Delta \boldsymbol{Q}_{n}^{\text {cond }}$. The operating point is represented through $P_{n}^{D G}, Q_{n}^{D G}, P_{n}^{D e m a n d}, Q_{n}^{\text {Demand }}, \boldsymbol{Q}_{n}^{\text {cond }}$ and is the outcome of marketclearing mechanism and DSO DRES and net-load forecasts. $\boldsymbol{P}_{\boldsymbol{n}}$ and $\boldsymbol{Q}_{\boldsymbol{n}}$ presented in (B.2) and (B.3) illustrate the active and reactive power flows in each node $n$ that come from the branches.

Regarding the constraints to which this optimization problem is subjected, (B.2) and (B.3) are responsible to ensure the active and reactive power balance. The voltage limits in each node $n$ are established through (B.4) and the voltage angle at the reference bus is set in (B.5). The discrete values that the capacitor banks and the OLTC can assume are specified in (B.6) and (B.7). Inequality constraints (B.8) and (B.9) refer to the direct and inverse branch flows limits. The remaining constraints are responsible to include in the problem formulation the flexibility band limits of each resource.

The decision variables, as already described in this manuscript, are the activated flexibilities within the corresponding limits and the voltage magnitude at the reference bus:

$$
\begin{aligned}
& \Delta P_{h}^{D G}, \Delta Q_{h}^{D G} \forall h \in N_{G} \\
& \Delta P_{i}^{\text {Demand }}, \quad \Delta Q_{i}^{\text {Demand }} \forall i \in N_{L} \\
& \Delta Q_{c}^{\text {cond }} \forall c \in N_{c} \\
& \Delta \text { OLTC }_{i j}^{t} \forall t \in T
\end{aligned}
$$

\section{References}

Al-digs, A., Chen, Y.C., 2016. Estimating feasible nodal power injections in distribution networks. In: Proceedings of the 2016 IEEE Power \& Energy Society Innovative Smart Grid Technologies Conference https://doi.org/10.1109/ISGT.2016. 7781244.

Bucher, M.A., Delikaraoglou, S., Heussen, K., Pinson, P., Andersson, G., 2015. On quantification of flexibility in power systems. In: Proceedings of the 2015 IEEE Eindhoven PowerTech doi:10.1109/PTC.2015.7232514.

Burchett, R.C., Happ, H.H., Vierath, D.R., 1984. Quadratically convergent optimal power flow. IEEE Trans. Power Appar. Syst. PAS-103, 3267-3275. doi:10.1109/ TPAS.1984.318568.

Carpentier, J., 1992. Contribution à l'étude du dispatching économique. Bull. Soc. Fr. Elect. 3, 431-447.

Eurelectric, 2015. Flexibility and aggregation - requirements for their interaction in the market. IEEE Trans. Power Syst. 30, 13. doi:10.1109/TPWRS.2014.2328865.

Gill, P.E., Murray, W., Saunders, M.A., Tomlin, J.A., Wright, M.H., 1986. On projected newton barrier methods for linear programming and an equivalence to Karmarkar's projective method. Math. Program 36, 183-209. doi:10.1007/ BF02592025.

Gonzaga, C.C., 1992. Path-following methods for linear programming. SIAM Rev. 34 167-224. doi:10.1137/1034048.

Haffner, S., Monticelli, A., Garcia, A., Romero, R., 2001. Specialised branch-and-bound algorithm for transmission network expansion planning. Gener. Transm. Distrib. IEE Proc. 148, 482-488. doi:10.1049/ip-gtd:20010502.

Heleno, M., Soares, R., Sumaili, J., Bessa, R.J., Seca, L., Matos, M.A., 2015. Estimation of the flexibility range in the transmission-distribution boundary. In: Proceedings of the 2015 IEEE Eindhoven PowerTech doi:10.1109/PTC.2015.7232524.

Hogan, P.M., Rettkowski, J.D., Bala, J.L.J., 2005. Optimal capacitor placement using branch and bound. In: Proceedings of the 37th Annual North American Power Symposium, 2005, pp. 84-89. doi:10.1109/NAPS.2005.1560506.

Karmarkar, N., 1984. A new polynomial-time algorithm for linear programming. Combinatorica 4, 373-395. doi:10.1007/BF02579150.

Krause, O., Lehnhoff, S., Handschin, E., Rehtanz, C., Wedde, H.F., 2009. On feasibility boundaries of electrical power grids in steady state. Int. J. Electr. Power Energy Syst. 31, 437-444. doi:10.1016/j.ijepes.2009.02.004 
ID: CAOR

[m5G ;June 14, 2017;7:22]

J. Silva et al./Computers and Operations Research 000 (2017) 1-11

11

Lu, W., Papalexopoulos, A., Piney, W.F., 1992. Discrete shunt control in a Newton optimal power flow. IEEE Trans. Power Syst. 7, 1509-1518. http://dx.doi.org/10. $1109 / 59.207375$.

Madureira, A.G., 2010. Coordinated and Optimized Voltage Management of Distributton Networks with Multi-Microgrids. Faculty of Engineering of University of Porto.

Marsten, R.E., Saltzman, M.J., Shanno, D.F., Pierce, G.S., Ballintijn, J.F., 1989. Amplementation of a dual affine interior point algorithm for linear programming. ORSA J. Compute. 1, 287-297. doi:10.1287/ijoc.1.4.287.

Mathieu, S., Ernst, D., Corn, B., 2016. Agent-based analysis of dynamic access ranges to the distribution network. In: Proceedings of the IEEE PES Innovative Smart Grid Technologies Conference Europe https://doi.org/10.1109/ISGTEurope.2016. 7856191.

Megiddo, N., 1989. Progress in Mathematical Programming. Springer New York, New York doi:10.1007/978-1-4613-9617-8_8.

Monticelli, A., Lu, W.H.E., 1992. Adaptive movement penalty method for the newton optimal power flow. IEEE Trans Power Syst 7, 334-342. doi:10.1109/59.141723.

Nosair, H., Bouffard, F., 2015. Flexibility envelopes for power system operatonal planning. IEEE Trans. Sustain Energy 6, 800-809. doi:10.1109/TSTE.2015. 2410760.

Nosair, H., Bouffard, F., 2016. Energy-centric flexibility management in power systerms. IEEE Trans. Power Syst. 31, 5071-5081. doi:10.1109/TPWRS.2015.2512990.

Polymeneas, E., Meliopoulos, S., 2016. Aggregate modeling of distribution systems for multi-period OPF. In: Proceedings of the 19th Power Systems Computation Conference doi:10.1109/PSCC.2016.7540987.

Pudjianto, D., Pudjianto, D., Ramsay, C., Ramsay, C., Strbac, G., Strbac, G., 2007. Virtull power plant and system integration of distributed energy resources. Renew. Power Gener. IET 1, 10-16. doi:10.1049/iet-rpg.

Saigal, R., 1995. Linear Programming: A Modern Integrated Analysis. Springer, US doi:10.1007/978-1-4615-2311-6.
Six, D., Ramos, A., Puente, E.R., 2014. Evaluation of Current Market Architectures and Regulatory Frameworks and The Role of DSOs 68. Deliverable D1.2 http: //www.evolvdso.eu/Home/Results

Stet, B., Marinho, J.L., Alsace, O., 1979. Review of linear programming applied to power system rescheduling. In: Proceedings of the IEEE Conference on Power Industry Computer Applications Conference, pp. 142-154. doi:10.1109/ PICA.1979.720058.

Piney, W.F., Bright, J.M., Demaree, K.D., Hughes, B.A., 1988. Some deficiencies in optimal power flow. IEEE Trans. Power Syst. 3, 676-683. doi:10.1109/59.192922.

Troy, N., Denny, E., O'Malley, M., 2010. Base-load cycling on a system with significant wind penetration. IEEE Trans. Power Syst. 25, 1088-1097. doi:10.1109/TPWRS. 2009.2037326.

Wan, Y., 2011. Analysis of Wind Power Ramping Behavior in ERCOT. National Renewable Energy Laboratory, Office of Energy Efficiency \& Renewable Energy, Golden, Colorado Report No.:NREL/TP-5500-49218. Contract No.:DE-AC36-08GO28308 doi:10.2172/1009681.

Zegers A, Brynner H. TSO-DSO interaction: An Overview of current interaction between transmission and distribution system operators and an assessment of their cooperation in Smart Grids. Seoul, Korea: International smart grid action network (ISGAN); 2014 September. ISGAN Discussion Paper Annex 6 Power T\&D Systems, Task 5.

Zhao, J., Zheng, T., Litvinov, E., 2016. A unified framework for defining and measuring flexibility in power system. IEEE Trans. Power Syst. doi:10.1109/TPWRS.2015. 2390038.

Zhao, L., Zhang, W., 2016. A geometric approach to virtual battery modeling of thenmostatically controlled loads. Proc. Am. Control Conf. 1452-1457. doi:10.1109/ ACC.2016.7525121, 2016-July.

Zimmerman, R.D., Murillo-Sanchez, C.E., Thomas, R.J., 2011. MATPOWER: steadystate operations, planning, and analysis tools for power systems research and education. IEEE Trans. Power Syst. 26, 12-19. doi:10.1109/TPWRS.2010.2051168.

Please cite this article as: J. Silva et al., The challenges of estimating the impact of distributed energy resources flexibility on the TSO/DSO

boundary node operating points, Computers and Operations Research (2017), http://dx.doi.org/10.1016/j.cor.2017.06.004 